\title{
SATISFACCIÓN DEL USUARIO EXTERNO QUE ACUDE AL SERVICIO DE EMERGENCIA DEL HOSPITAL REGIONAL DE ICA. AÑO 2018
}

\author{
Satisfaction Of The External User Going To The Emergency Service Of The Regional \\ Hospital Of Ica. Year 2018
}

\section{Alexandra Stefany Napa Pisconte ${ }^{1 a}$}

${ }^{1}$ Facultad de Enfermería. Universidad Nacional San Luis Gonzaga de Ica. Ica, Perú.

a Lic. En Enfermería

\section{RESUMEN}

Objetivo: Determinar la satisfacción del usuario externo que acude al servicio de emergencia del Hospital Regional de Ica, Año 2018. Material y Métodos: La investigación con carácter Descriptivo, criterio de análisis Cualicuantitativa y de Corte transversal, con una muestra dado al presupuesto y el tiempo de 100 usuarios externos que acuden al servicio de Emergencia del Hospital Regional de Ica, en el año 2018. El instrumento que se aplicó es la Encuesta Servqual modificada compuesta con 22 ítems. Resultados: Se identificó el grado de satisfacción del usuario externo del servicio de emergencia, detallándose según dimensiones las siguientes cifras de satisfacción: fiabilidad $22,00 \%$, capacidad de respuesta $25,75 \%$, seguridad $23,75 \%$, empatía $23,80 \%$ y aspectos tangibles $24,25 \%$; por otro lado encontramos las siguientes cifras de insatisfacción: fiabilidad $78.00 \%$, capacidad de respuesta $74,25 \%$, seguridad $76,25 \%$, empatía $76,20 \%$ y aspectos tangibles $75,25 \%$. Conclusiones: Ante el análisis realizado se obtuvo como resultado global de satisfacción un $23,82 \%$ y de insatisfacción se obtuvo como un $76,18 \%$.

Palabras Claves: Satisfacción del usuario externo, Servicio de Emergencia

\section{SUMMARY}

Objective: "Determine the satisfaction of the external user who goes to the emergency department of the Regional Hospital of Ica, year 2018". Material and Methods: The descriptive research, the qualitative criteria of quantitative and cross-sectional analysis, with a sample given to the budget and the time of 100 external users who go to the Emergency Department of the Regional Hospital of Ica, in 2018. The instrument was applied Modified Servqual Survey composed of 22 items. Results: The degree of satisfaction of the external user of the emergency service was identified, detailing the following satisfaction figures according to the dimensions: reliability $22.00 \%$, responsiveness $25.75 \%$, security $23.75 \%$, empathy $23.80 \%$ and tangible aspects $24.25 \%$; On the other hand, we find the following dissatisfaction figures: reliability $78.00 \%$, responsiveness $74.25 \%$, security $76.25 \%$, empathy $76.20 \%$ and tangible aspects $75.25 \%$. Conclusions: $23.82 \%$ was obtained as a result of the analysis performed, and $76.18 \%$ was obtained as dissatisfaction.

Key words: External user satisfaction, Emergency Service 


\section{INTRODUCCIÓN}

En el Perú, el Ministerio de Salud (MINSA), cumple el papel de autoridad sanitaria nacional, administrando el Sistema de Salud. A fines del año 2013, surgió SUSALUD ("Superintendencia Nacional de Salud"), institución cuya función es supervisar todas las organizaciones sanitarias; estas dos entidades tienen responsabilidades compartidas de gestión en la política de salud del país (1).

Según las cifras que mostró el Instituto Nacional de Estadística e Informática (INEI: 2015), se observa que desde 2004 al año 2014 hubo un significativo cambio en los motivos de las personas, que, a pesar de requerir atención sanitaria, no acudían a estos servicios, demostrándose en 2004 que el motivo por el cual decidían no acudir a citas médicas fue por falta de dinero en un $24,7 \%$, después de una década esta cifra logró reducirse a un 8,5\% (2).

No obstante, otros obstáculos para el acceso son: distancia geográfica, desconfianza y las demoras, éstas aumentaron en el período ya mencionado, de un $12,3 \%$ a $17 \%$. Además, de las personas no aseguradas, falta de tiempo y el pésimo trato en la atención, crecieron en el mismo período de $11 \%$ a $23,9 \%$. La problemática económica del acceso a los servicios sanitarios se desplazó al tema de la calidad del servicio (2).

Esta oportunidad, hablamos del Hospital Regional de Ica (H.R.I.), donde desarrolla los pilares de: promoción, prevención, recuperación, rehabilitación de salud y de gestión.

Para ello es valioso realizar investigaciones científicas que permitan aportar información sobre la atención brindada, siendo de gran beneficio para comprender el comportamiento del paciente; facilitando el desarrollo de una buena relación con él; contribuyendo a identificar, analizar y evaluar las situaciones que genere el proceso de atención, para establecer y ejecutar acciones de manera preventiva en caso fueran requeridas, mejorando así la atención.

Este estudio posee el objetivo primordial de Determinar la satisfacción del usuario externo en el servicio de emergencia del Hospital Regional de Ica en el año 2018.

Por lo tanto, a través del presente estudio, se plantea que con una intervención oportuna y de calidad se puede obtener así resultados que permitan disminuir las complicaciones al momento de la atención y reduciendo la tasa de mortalidad en dicho establecimiento de salud.

Indudablemente, nuestro Perú, es un país con un considerable crecimiento económico en América Latina. Siendo evidente el descenso progresivo de la pobreza repercutiendo en un progreso en salud. Para seguir con la mejora en los indicadores establecidos, es necesario conservar el esfuerzo para cubrir la cobertura y el acceso de salud (1).

\section{MATERIALES Y MÉTODOS}

Esta investigación es de tipo cualicuantitativa, de método descriptivo, no experimental y de corte transversal. El tamaño muestral fue constituido por 100 usuarios atendidos en el Servicio de Emergencia del Hospital Regional de Ica. 
Este estudio utilizó como instrumento la Encuesta Servqual Modificado adaptado y validado a la realidad del país (3), dividida en 5 dimensiones, abarcando dos campos y con 22 ítems cada uno, primer campo "expectativa" y segundo campo "percepciones".

Siendo la demanda de usuarios una característica distintiva del servicio de emergencia, se estableció un período de 15 días de aplicación del instrumento, permitiendo así una adecuada recolección de datos. Para el análisis de los datos obtenidos se hizo uso de dos programas: Microsoft Excel, el cual permitió la creación de la base de datos para su posterior procesamiento y el programa estadístico SPSS 24 para la consolidación de datos.

Los resultados del análisis ejecutado de los datos, fueron expresados en cifras de porcentajes de acuerdo a la consolidación establecida, por cada condición, pregunta, criterio de calidad y nivel de satisfacción.

Se ha considerado que para el nivel de satisfacción del usuario, el resultado de la sustracción entre percepción $(P)$ y expectativa (E); para lo cual, sí contamos con un resultado positivo responde al logró de la satisfacción, por lo contrario, si se obtiene un valor negativo estamos ante una insatisfacción del usuario.

Acerca la interpretación de valores que se han obtenido, a través del aplicativo de uso se consideró respecto al nivel de satisfacción: tabla de las condiciones de encuestados, tablas respuesta a los ítems y criterio, una matriz de mejora por criterio.

En esta última mención "matriz de mejora", es preciso mencionar que tiene una característica de prioridad según el color relativo al porcentaje obtenido de insatisfacción: mayor de $60 \%$ (rojo - "por mejorar"); entre 40 y $60 \%$ (amarillo - "en proceso"); y menos de $40 \%$ (verde - "aceptable") (4).

\section{RESULTADOS}

En la Tabla $\mathrm{N}^{\circ} 1$, podemos observar los datos generales del encuestado divididos en seis características: en condición del encuestado obtenemos un $50,3 \%$ como usuario y un $49,7 \%$ como acompañantes; en edad divididos según grupos etários jóvenes de 18-29, adultos 30 - 59 y adultos mayores de $60 \mathrm{a}+$ obteniendo un 19,9\%, 51,9\% y $28,2 \%$ respectivamente; en sexo obtenemos un $38,1 \%$ en sexo masculino y un $61,9 \%$ en sexo femenino; en nivel de estudio obtenemos un $1,7 \%$ en analfabeto, $22,1 \%$ en primaria, $47,5 \%$ secundaria, $9,9 \%$ superior técnico y $18,8 \%$ superior universitario; en tipo de seguro según SIS, SOAT, Ninguno y otro hallándose un $68 \%, 0,6 \%, 24,9 \%$ y $6,6 \%$ respectivamente; según tipo de usuario obtenemos un $30,9 \%$ en nuevo y $69,1 \%$ como continuador. 
Napa A.

Tabla $N^{\circ} 1$ datos generales de usuarios encuestados DEL SERVICIO DE EMERGENCIA DEL H.R.I. AÑO 2018

\begin{tabular}{ccc}
\hline CONDICIÓN DEL ENCUESTADO & $\mathbf{N}^{\circ}$ & $\%$ \\
\hline Usuario & 50 & 50,3 \\
Acompañante & 50 & 49,7 \\
\hline EDAD & $\mathbf{N}^{\circ}$ & $\%$ \\
\hline Jóvenes 18 - 29 & 20 & 19,9 \\
Adulto 30 - 59 & 52 & 51,9 \\
Adultos mayores 60 a + & 28 & 28,2 \\
\hline SEXO & $\mathbf{N}^{\circ}$ & $\%$ \\
\hline Masculino & 38 & 38,1 \\
Femenino & 62 & 61,9 \\
\hline NIVEL DE ESTUDIO & $\mathbf{N}^{\circ}$ & $\%$ \\
\hline Analfabeto & 2 & 1,7 \\
Primaria & 22 & 22,1 \\
Secundaria & 47 & 47,5 \\
Superior Técnico & 10 & 9,9 \\
Superior Universitario & 19 & 18,8 \\
\hline TIPO DE SEGURO & $\mathbf{N}^{\circ}$ & $\%$ \\
\hline SIS & 68 & 68,0 \\
SOAT & 1 & 0,6 \\
Ninguno & 25 & 24,9 \\
Otro & 7 & 6,6 \\
\hline TIPO DE USUARIO & $\mathbf{N}^{\circ}$ & $\%$ \\
\hline Nuevo & 31 & 30,9 \\
Continuador & 69 & 69,1 \\
\hline
\end{tabular}

Fuente: Base de datos - Cuestionario

En la tabla №2 se estableció la satisfacción de los usuarios atendidos en emergencia relacionado a sus percepción (P) y expectativa (E), obteniendo las siguientes cifras de acuerdo a cada pregunta reflejando la satisfacción de (P menos $\mathbf{E})$ : $21 \%, 21 \%, 28 \%, 28 \%, 12 \%, 21 \%, 26 \%$, $38 \%, 18 \%, 24 \%, 26 \%, 28 \%, 17 \%, 22 \%$, $22 \%, 27 \%, 27 \%, 21 \%, 30 \%, 15 \%, 26 \%$,
$26 \%$ y como resultado un promedio de satisfacción $23,82 \%$. Según las cifras de insatisfacción se obtuvo como promedio total $76,18 \%$. En percepción, observamos que se obtuvo un promedio mayor en la pregunta $(P) 19$ con 6,56 y la cifra menor en la pregunta 8 con 4,83 . En expectativa, vemos el promedio oscilando en 6,98 y 6,99 . 
Tabla N ${ }^{\circ} 2$ SATISFACCIÓN DE LOS USUARIOS EXTERNOS ENCUESTADOS EN EL SERVICIO DE EMERGENCIA DEL H.R.I. RELACIONADO A SUS PERCEPCIONES Y

EXPECTATIVAS AÑO 2018

\begin{tabular}{ccccc}
\hline P - E & SATISFECHO & INSATISFECHO & PERCEPCIÓN & EXPECTATIVA \\
\hline P1 & $21 \%$ & $79 \%$ & 4,92 & 6,98 \\
P2 & $21 \%$ & $79 \%$ & 5,09 & 6,98 \\
P3 & $28 \%$ & $72 \%$ & 5,47 & 6,98 \\
P4 & $28 \%$ & $72 \%$ & 5,26 & 6,98 \\
P5 & $12 \%$ & $88 \%$ & 4,87 & 6,99 \\
P6 & $21 \%$ & $79 \%$ & 5,22 & 6,99 \\
P7 & $26 \%$ & $74 \%$ & 5,4 & 6,98 \\
P8 & $38 \%$ & $62 \%$ & 4,83 & 6,99 \\
P9 & $18 \%$ & $82 \%$ & 5,47 & 6,99 \\
P10 & $24 \%$ & $76 \%$ & 5,47 & 6,99 \\
P11 & $26 \%$ & $74 \%$ & 5,25 & 6,99 \\
P12 & $28 \%$ & $72 \%$ & 5,28 & 6,98 \\
P13 & $17 \%$ & $83 \%$ & 5,06 & 6,98 \\
P14 & $22 \%$ & $78 \%$ & 5,46 & 6,99 \\
P15 & $22 \%$ & $78 \%$ & 5,28 & 6,99 \\
P16 & $27 \%$ & $73 \%$ & 5,42 & 6,98 \\
P17 & $27 \%$ & $73 \%$ & 5,36 & 6,99 \\
P18 & $21 \%$ & $79 \%$ & 5,19 & 6,98 \\
P19 & $30 \%$ & $70 \%$ & 6,56 & 6,99 \\
P20 & $15 \%$ & $85 \%$ & 5,03 & 6,99 \\
P21 & $26 \%$ & $74 \%$ & 5,41 & 6,99 \\
P22 & $26 \%$ & $74 \%$ & 5,37 & 6,99 \\
\hline PROMEDI0 & $\mathbf{2 3 , 8 2 \%}$ & $\mathbf{7 6 , 1 8 \%}$ & $\mathbf{5 , 3}$ & $\mathbf{6 , 9 9}$ \\
\hline
\end{tabular}

Fuente: Base de datos - Cuestionario

En la Tabla $N^{\circ} 3$, se identificó el grado de satisfacción de usuarios externos en emergencia de acuerdo a la aplicación del instrumento ", se detalla según dimensiones las siguientes cifras de satisfacción: fiabilidad 22,00\%, capacidad de respuesta 25,75\%, seguridad $23,75 \%$, empatía $23,80 \%$ y aspectos tangibles $24,25 \%$, asimismo hallamos cifras de insatisfacción de: fiabilidad 78,00\%, capacidad de respuesta $74,25 \%$, seguridad $76,25 \%$, empatía $76,20 \%$ y aspectos tangibles $75,25 \%$

TABLA N ${ }^{\circ} 3$ GLOBAL DIMENSIONES DE LA SATISFACCIÓN DE LOS USUARIOS EXTERNOS QUE ACUDEN AL SERVICIO DE EMERGENCIA DEL H.R.I. AÑO 2018

\begin{tabular}{ccc}
\hline DIMENSIONES & SATISFECHO (+) & INSATISFECHO (-) \\
\hline FIABILIDAD & $2200 \%$ & $78,00 \%$ \\
CAPACIDAD DE RESPUESTA & $25,75 \%$ & $74,25 \%$ \\
SEGURIDAD & $23,75 \%$ & $76,25 \%$ \\
EMPATÍA & $23,80 \%$ & $76,20 \%$ \\
ASPECTOS TANGIBLES & $24,25 \%$ & $75,75 \%$ \\
\hline PROMEDIO TOTAL & $\mathbf{2 3 , 9 1 \%}$ & $\mathbf{7 6 , 0 9 \%}$ \\
\hline
\end{tabular}

Fuente: Base de datos - Cuestionario 
En la tabla $\mathrm{N}^{\circ} 4$, nuestra matriz de mejora, se analizó las 22 preguntas que conforman la encuesta servqual, según los parámetros establecidos en la guía aplicativa referencial MINSA, respecto al procesamiento de los datos registrados, ante el análisis se obtuvo como resultado en su totalidad cifras de insatisfacción mayores al $60 \%$ y de color rojo, es decir, que se requiere de una mejora abarcando todos los ítems en sus 5 criterios de calidad 0 dimensiones. Podemos observar que la pregunta 5 (relacionado al abastecimiento de la farmacia sobre los medicamentos) alcanzó un 88\% de insatisfacción, siendo está la cifra mayor. Por lo contrario, hay un triple empate en la cifra que sería menor en la matriz de mejor, pero no libre de una mejoría que correspondería a la pregunta 3 (referente sí el médico estuvo a cargo de su atención), la pregunta 4 (sobre sí el médico tuvo la comunicación necesaria con el usuario para informarle sobre la situación de salud) y la pregunta 12 (relacionado a confirmar si es que el médico realizado un examen físico previo a la atención) con $72 \%$ de insatisfacción.

TABLA N 4 MATRIZ DE MEJORA DE USUARIOS EXTERNOS ENCUESTADOS EN EL SERVICIO DE EMERGENCIA DEL H.R.I. AÑO 2018

\begin{tabular}{|c|c|c|c|c|c|}
\hline \multirow[t]{2}{*}{ DIMENSIÓN } & \multirow[t]{2}{*}{ PREGUNTAS } & \multicolumn{2}{|c|}{$\begin{array}{c}\text { SATISFECHOS } \\
(+)\end{array}$} & \multicolumn{2}{|c|}{$\begin{array}{c}\text { INSATISFECHOS } \\
(-)\end{array}$} \\
\hline & & $\mathbf{n}$ & $\%$ & $\mathbf{n}$ & $\%$ \\
\hline \multirow{5}{*}{ FIABILIDAD } & P01 & 21 & $21 \%$ & 79 & $79 \%$ \\
\hline & P02 & 21 & $21 \%$ & 79 & $79 \%$ \\
\hline & P03 & 28 & $28 \%$ & 72 & $72 \%$ \\
\hline & P04 & 28 & $28 \%$ & 72 & $72 \%$ \\
\hline & P05 & 12 & $12 \%$ & 88 & $88 \%$ \\
\hline \multicolumn{2}{|c|}{ PROMEDIO FIABILIDAD } & & 22 & & 78 \\
\hline \multirow{4}{*}{ CAPACIDAD DE RESPUESTA } & $\mathrm{P} 06$ & 21 & $21 \%$ & 79 & $79 \%$ \\
\hline & P07 & 26 & $26 \%$ & 74 & $74 \%$ \\
\hline & P08 & 38 & $38 \%$ & 62 & $62 \%$ \\
\hline & P09 & 18 & $18 \%$ & 82 & $82 \%$ \\
\hline \multicolumn{2}{|c|}{ PROMEDIO CAPACIDAD DE RESPUESTA } & & $26 \%$ & & $74 \%$ \\
\hline \multirow{4}{*}{ SEGURIDAD } & $\mathrm{P} 10$ & 24 & $24 \%$ & 76 & $76 \%$ \\
\hline & P11 & 26 & $26 \%$ & 74 & $74 \%$ \\
\hline & P12 & 28 & $28 \%$ & 72 & $72 \%$ \\
\hline & $\mathrm{P} 13$ & 17 & $17 \%$ & 83 & $83 \%$ \\
\hline \multicolumn{2}{|c|}{ PROMEDIO SEGURIDAD } & & $24 \%$ & & $76 \%$ \\
\hline \multirow{5}{*}{ EMPATÍA } & $\mathrm{P} 14$ & 22 & $22 \%$ & 78 & $78 \%$ \\
\hline & P15 & 22 & $22 \%$ & 78 & $78 \%$ \\
\hline & P16 & 27 & $27 \%$ & 73 & $73 \%$ \\
\hline & P17 & 27 & $27 \%$ & 73 & $73 \%$ \\
\hline & P18 & 21 & $21 \%$ & 79 & $79 \%$ \\
\hline \multicolumn{2}{|c|}{ PROMEDIO EMPATIA } & & $24 \%$ & & $76 \%$ \\
\hline \multirow{4}{*}{ ASPECTOS TANGIBLES } & P19 & 30 & $30 \%$ & 70 & $70 \%$ \\
\hline & P20 & 15 & $15 \%$ & 85 & $85 \%$ \\
\hline & P21 & 26 & $26 \%$ & 74 & $74 \%$ \\
\hline & P22 & 26 & $26 \%$ & 74 & $74 \%$ \\
\hline \multicolumn{2}{|c|}{ PROMEDIO ASPECTOS TANGIBLES } & & $24 \%$ & & $76 \%$ \\
\hline \multicolumn{2}{|c|}{ Porcentaje Total } & & $24 \%$ & & $76 \%$ \\
\hline
\end{tabular}




\section{DISCUSIÓN}

Respecto a datos generales de los usuarios externos participantes del estudio, aplicada a un total de 100 encuestados en el H.R.I., se identificó que el $50,3 \%$ son usuarios y el otro $49,7 \%$ son acompañantes (en la tabla $N^{\circ} 1$ ), presentándose una similitud con las cifras obtenidas en el Hospital Regional de Docentes de Cajamarca (2017) (5); con una muestra total de 374 usuarios encuestados, de los cuales $50 \%$ son usuarios y $50 \%$ acompañantes.

Las edades oscilan entre 18 a 84 años, teniendo en jóvenes un $19,9 \%$ de 18 a 29 años, adultos un equivalente a $51,9 \%$ de 30 a 50 años y un $28,2 \%$ para aquellos adultos mayores de 60 años a más. Tomando en cuenta como mayor porcentaje de usuarios atendidos se encuentra dentro del rango de los adultos.

Haciendo mención a las cifras obtenidas acerca del sexo de los encuestados, el Hospital Regional de Docentes de Cajamarca (2017) indica que hubo un $31,55 \%$ de usuarios masculinos, mientras que el $68,45 \%$ era femenino. En comparación con las cifras obtenidas en el presente trabajo, el $38,1 \%$ es la parte masculina, mostrando una diferencia ya que el $61,9 \%$ representa la parte femenina. Demostrando que, en su mayoría, se encuestó a la población femenina. Ya que, por lo general, es la población más recurrente para recibir un servicio de salud.

Continuando con las características de los encuestados, acerca del nivel de instrucción o estudio, el Hospital Regional Docente de Cajamarca (2017) resolvió que el $2,95 \%$ son usuarios que no saben leer ni escribir, $8,83 \%$ son aquellos usuarios que pudieron estudiar el nivel primario, el $45,73 \%$ optaron por terminar sus estudios secundarios, el $18,45 \%$ son aquellos que han estudiado alguna carrera técnica y el $23,80 \%$ lograron una carrera profesional. Mostrando una clara y evidente diferencia al comparar con los resultados obtenidos, considerando los cinco niveles de estudio: analfabetismo, nivel primario, nivel secundario, superior técnico y superior universitario; teniendo como cifras: $1,7 \%, 22,1 \%, 47,5 \%, 9,9 \%$ y $18,8 \%$ respectivamente. La mayor cantidad de los usuarios encuestados son aquellos que alcanzaron el nivel de estudio secundario.

Los pacientes, según los resultados que arrojaron las encuestas, cuentan con algún tipo de seguro, predominando el SIS, ya que es el Estado quien lo brinda. En el Hospital Regional Docente de Cajamarca (2017) equivale el $77,54 \%$ mientras que en el H.R.I. (2016) fue de $86,8 \%$. Se presentó una gran diferencia de los usuarios que contaban con el SIS, ya que en el año 2018 se obtuvo que el $68,0 \%$ contaba con ese tipo de seguro. Por otro lado, la cantidad de los usuarios que no asegurados es de $24,9 \%$.

Para terminar con las condiciones del encuestado, se obtuvo que el $30,9 \%$ son usuarios nuevos, pacientes que recientemente están siendo atendidos. Por otro lado, notable diferencia, el $69,1 \%$ son pacientes que siguen recibiendo algún tipo de servicio de salud por alguna complicación presentada. Caso contrario son los resultados que obtuvo el Hospital Regional Docente de Cajamarca (2017), $\quad 75,13 \%$ y $24,87 \%$ respectivamente.

De acuerdo a los valores obtenidos respecto al nivel de satisfacción del usuario externo según percepción y expectativa de los 22 ítems de la 
ENCUESTA SERVQUAL, en la Tabla $\mathrm{N}^{\circ} 2$, la percepción de los usuarios encuestados presentó un promedio menor de 4,83 (P8: que consiste en la atención rápida para la toma de exámenes radiológicos), un promedio mayor de 6,56 (P19: que consiste sí le parece adecuada la señalización del servicio) y una media de 5,28; las expectativas oscilan en un promedio de 6,98 y 6,99 . Los promedios generales son de 5,30 y 6,99 respectivamente.

Con respecto a las cifras obtenidas en el Hospital Regional de Cajamarca (2017), nos indica que la satisfacción tuvo un tope máximo equivalente al mayor promedio de 60,2\% (P22: consiste en la limpieza y comodidad del servicio) y un promedio menor de $7 \%$ (P7: consiste en la atención recibida en Laboratorio del servicio). Mientras que la insatisfacción tiene un pico de promedio mayor de $93,05 \%$ (P7) y un promedio menor de $39,84 \%$ (P22). Podremos darnos cuenta que, en el Hospital Regional Docente de Cajamarca, el punto fuerte de satisfacción que indican los resultados tras los datos obtenidos de los encuestados es que los ambientes se encuentran adecuados, resaltando la limpieza y comodidad que brindan en dicho establecimiento. Sin embargo, se encontraron completamente insatisfechos con la atención que recibieron al momento de realizarse los análisis en el laboratorio, ya que, pese a la urgencia, no era una atención rápida ni procuraban hacerlo.

Por otra parte, los datos obtenidos en este trabajo arrojaron que, en el H.R.I. la satisfacción es representada con un promedio general porcentual de $23,82 \%$, teniendo una cifra menor de 12\% (P5: consiste en el abastecimiento de la farmacia), cifra mayor de $38 \%$ (P8: consiste en la atención para la toma de exámenes radiológicos) y una media de 24\% (P10: consiste en el tiempo brindado por parte del médico para aclarar posibles dudas sobre la situación del usuario atendido). Para lo cual las cifras de satisfacción no son las deseadas, de esta manera nos reflejan que los usuarios se encontraban ligeramente satisfechos con la rapidez de la atención que les brindaban al momento de realizarse los exámenes radiológicos, seguidamente con el tiempo que el médico le brindaba explicándole el estado del usuario.

Prosiguiendo con las cifras de la insatisfacción son relativas a las ya mencionadas anteriormente, por lo tanto, el promedio general de insatisfacción es de $76,18 \%$, la cifra mayor, menor y media son de un $88 \%$ $\begin{array}{lllll}(\mathrm{P} 5), & 62 \% & \text { (P8) y } & 76 \% & \text { (P10) }\end{array}$ respectivamente. Presentando un notable nivel de insatisfacción acerca del abastecimiento que contaba el área de farmacia, ya que no podían adquirir de inmediato los medicamentos que eran recetados por el servidor de salud.; siendo preocupante el gran número de pacientes que no podían conseguirlos por otros medios, pese a la situación en la que se encontraban. Además del poco tiempo brindado por el médico para establecer la seguridad en el paciente y la demora para los exámenes radiológicos.

Con respecto a las dimensiones de la satisfacción del usuario externo, nos presenta el nivel de satisfacción e insatisfacción de cada dimensión se observa en la Tabla $N^{\circ} 3$. Fiabilidad con una satisfacción de 22,00\%, mientras que la mayoría indicaron estar insatisfechos, siendo ellos el $78,00 \%$, ya que argumentaron que no fueron atendidos como ellos lo esperaban. Capacidad de Respuesta, el 25,75\% de usuarios indicaron satisfacción con este criterio, y un $74,25 \%$ indicaron estar insatisfechos ya que no contaron con una atención rápida y oportuna. Seguridad, el $23,75 \%$ de los usuarios 
atendidos indicaron que el personal de salud les brindó la confianza necesaria para ser atendidos de forma adecuada, por lo contrario, el $76,25 \%$ no contaron con la confianza a plenitud por parte del equipo de salud que les brindaron la atención correspondiente. Empatía, el $23,80 \%$ de los usuarios atendidos indicaron que los servidores de salud que les atendieron comprendían su situación y trataban de resolver cualquier problema que se presentaba en su atención; sin embargo, el 76,20\% muestra insatisfacción los usuarios sobre el apoyo que les pudieron brindar el personal de salud más allá de cumplir con sus deberes. Aspectos Tangibles, el $24,25 \%$ estaban satisfechos con la infraestructura, personal y equipos que se encontraban y eran requeridos para su atención, pese a ello, el 75,75\% lamentablemente se encontraron totalmente insatisfechos ya que para ellos no cumplían con las expectativas que tenían acerca del establecimiento, personal de salud y hasta del estado de los equipos mismos.

Por nociones de estudio, se está tomando en cuenta en esta parte de la investigación al antecedente nacional del Hospital Regional Docente de Cajamarca con el propósito de comparar los resultados siendo acorde a la realidad, considerando los puntos más relevantes para dicho análisis.

El antecedente en mención presentó como nivel de satisfacción, encontrando la dimensión seguridad con un $37,50 \%$ y dejando en claro una insatisfacción del $75,40 \%$ con respecto a la capacidad de respuesta. Los cálculos generales nos demuestran que el $31,30 \%$ de los usuarios encuestados se encontraron satisfechos y el $68,70 \%$ insatisfechos.

Interpretando podemos llegar a la siguiente conclusión, el punto fuerte del establecimiento de salud en donde este trabajo se realizó abarca la dimensión de la Capacidad de Respuesta, demostrando que los servidores de salud tienden a brindar su plena disposición en otorgar una atención adecuada y oportuna. Sin embargo, se quedó demostrado que la mayor deficiencia de dicha institución es acerca de la Fiabilidad, ya que los encuestados argumentaron que la atención que les brindaron no cumplía el objetivo principal, el de restablecer el estado óptimo de salud del paciente.

\section{CONCLUSIONES}

Las conclusiones están ligadas a los objetivos planteados, tomando en cuenta los puntos más importantes, por tal, llegamos a las siguientes:

- Se mostró en una gran mayoría de insatisfacción con la atención que les brindó el personal de la institución hospitalaria, sólo el 23,91\% demostraron satisfacción con el servicio que les brindó los profesionales de la institución hospitalaria.

- Respecto a la condición del usuario el $50,3 \%$ son pacientes que reciben el servicio de salud, de éstos en su mayoría son adultos con un porcentaje de $51,9 \%$, de los cuales el $61,9 \%$ nos indica que el sexo predominante es femenino. Demostrando que el nivel de estudio por lo general es el secundario con un $47,5 \%$, predominando el tipo de seguro el SIS con el 68,0\%. Por lo que los usuarios son recurrentes en su mayoría con un $69,1 \%$.

- La percepción de los usuarios es menor en comparación con las expectativas, resultando que el $23,82 \%$ de usuarios quedaron satisfechos con el servicio que les brindaron, mientras que los demás, 
equivalente al $76,18 \%$, indicaron estar insatisfechos.

- Respecto a criterios de calidad, se utilizaron cinco dimensiones, de los cuales se obtuvo las siguientes cifras de satisfacción, el mayor porcentaje corresponde a capacidad de respuesta con un $25,75 \%$, seguidamente estuvieron de acuerdo con los elementos tangibles el $24,25 \%$. El $23,80 \%$ de los usuarios indicaron casi simultáneamente que percibieron empatía tras su condición, con diferencia de una brecha mínima, la seguridad en su atención se determinó con un porcentaje de $23,75 \%$ y respecto a la fiabilidad del servicio un porcentaje menor de $22,00 \%$.

\section{Correspondencia:}

Lic. Alexandra Stefany Napa Pisconte

Correo electrónico:

stefany_acuario_1997@hotmail.com

\section{REFERENCIAS BIBLIOGRÁFICOS}

1. Lazo O, Alcalde J, Espinosa 0. Sistema de salud en Perú. $1^{\circ}$ ed. Perú: REP S.A.C; 2016: pp.13,37, 68,69

2. Arroyo J, Velásquez A, Céspedes S. Malo M., Pedroza J. Et al. La Salud Hoy: Problemas y Soluciones. $1^{\circ}$ ed. Perú: Ciento Uno Estudio Gráfico E. I. R. L.; 2015 p.17
3. Cabello E, Chirinos J. Validación y aplicabilidad de encuestas Servqual Modificadas para medir la satisfacción de usuarios externos en servicio de salud. Rev. Med Hered .2012; 23(2):88-95. (Internet) Lima, Perú; 2012. (Consultado: 14 de octubre 2018) Disponible en: http://www.scielo.org.pe/pdf/rmh/v2 3n2/v23n2ao2.pdf

4. Guerra R, Cabello E. Guía Técnica para la Evaluación de la Satisfacción del Usuario Externo en los Establecimientos y Servicios Médicos de Apoyo. RM № 5272011/MINSA. Dirección de Calidad en Salud. DCS-DGSP-MINSA. (Internet).Lima, Perú. (Consultado: 18 de agosto 2018) Disponible en: ftp://ftp2.minsa.gob.pe/descargas/c alidad/normas/documentos/doc Sa tisfaccion usu externo.pdf

5. Julcamoro M, Terán A. Nivel de satisfacción del usuario externo. servicio de emergencia del Hospital Regional docente de Cajamarca. 2017. Tesis Pregrado. Cajamarca, Perú. Facultad de Enfermería. Universidad Privada Antonio Guillermo Urrelo; 2017. pp.3,4,8

Recibido: 20/08/2019

Aprobado para publicación: 30/11/2019 